\title{
FoxfI and SP-C contains a cell-type-specific transcriptional activation domain and is expressed in mouse lungs
}

\begin{abstract}
Lung development in the mammal carries series of steps starting from simple lung buds to the elaborately branched organ that fulfil the vital function of gaseous exchange. Strict transcriptional control is essential for lung development. To screen the genes responsible for the regional growth and patterning we used RNA Insitu hybridization to identify genes that show restricted expression patterns in the embryonic lung. During organogenesis, the expression of Foxf1 becomes restricted to the splanchnic mesoderm, which provides the mesenchymal cells of the intestinal tract and gut derivates such as lungs and liver. Surfactant is a lipoprotein substance that is synthesized and secreted by alveolar type II epithelial cells and acts to reduce surface tension at the air-alveolar interface. SP-C a 5kDa, hydro-phobic, surfactant-associated protein. We used the technique of in situ hybridization to localize SP-C mRNA in mice lung tissue. SP-C mRNA was present in all of the epithelial cells of the prealveolar region of day 13 gestational age mice lung tissue. Our data are suggestive that SP-C is present in fetal lung prealveolar epithelial cells much earlier in gestation than are differentiated, surfactant-producing alveolar type II cells.
\end{abstract}

Keywords: lung development, mice, foxf1, SP-C, epithelial, mesenchyma cells, mesenchyme, epithelium, in situ hybridization, RNA
Volume 4 Issue 2 - 2016

Satheesh Natarajan,' Peter Carlsson'
'Department of Molecular Biology, Göteborg University, Sweden
'Department of Molecular Biology, Göteborg University, Sweden

Correspondence: Satheesh Natarajan, Department of Molecular Biology, Göteborg University, The Lundberg Laboratory, Box 462, SE-405 30,Göteborg, Sweden, Email satheesh.sahana@gmail.com

Received: October 13, 2016 | Published: October 28, 2016
Abbreviations: SHH, sonic hedgehog; FGF, fibroblast growth factor; BMP, bone morphogenetic protein

\section{Introduction}

For the survival starting from birth the mammalian lung is very essential and a prominent branching organ, with an endodermderived epithelium supported by a mesoderm-derived mesenchyme. In the mature lung, the epithelial tubules assume the shape of a treelike structure, where the proximal portion forms the conducting airways and the distal portion forms the gas-exchanging alveoli. The mesenchyme consists primarily of the vasculature, smooth muscles, and tracheal or bronchial cartilage that forms intimate contacts with the epithelial tree and are also critical for respiration. ${ }^{1}$ The five stages of the mice lung development are embryonic bud initiation (embryonic day, or E9.0-E11.5), pseudo glandular (E11.5-E16.5), canalicular (E16.5-E17.5), saccular (E17.5-P5), and alveolar (P5P28) stages. ${ }^{2} \mathrm{Nkx} 2-1$ in a small group of ventral foregut endoderm cells at E9.0 is the first sign of respiratory specification is marked by the expression of a transcription factor encoding gene. ${ }^{3}$ Signals from the prospective lung mesenchyme are critical for this specification. ${ }^{4}$ The primary lung buds is observed at E9.5, from E9.5-E11.5, these buds elongate and undergo secondary branching to establish the basis of one left lung lobe and four right lung lobes. The bulk of branching morphogenesis occurs during the pseudoglandular stage, and follows a strict program. ${ }^{5}$ Toward the end of this stage and continuing into the canalicular stage, the conducting airway epithelium differentiates into specialized cell types, including Clara and ciliated cells. ${ }^{6}$ Drastic changes occur in the saccular stage as the lung transitions from being fluid filled to air breathing. The distal epithelium undergoes saccular branching and subdivides into primary alveolar sacs that allow for gas exchange. Meanwhile, the vascular network surrounding the alveolar sacs remodels to facilitate oxygen uptake. Within the distal epithelium, type II pneumocytes mature and start to produce surfactants, a protein and lipid mixture that lowers surface tension within the lung and allows lung expansion at birth. ${ }^{7}$ The expression of channel proteins also increases at birth, leading to rapid clearing of the fluid from the lung. While the lung can carry out gas-exchange at the end of saccular stage, it only contains approximately $5 \%$ of the final alveolar surface area. During alveologenesis, thousands of secondary septae rises from the walls of the primary alveolar sac into the lumen; each serving as a new surface for gas-exchange. ${ }^{8}$ Transcription factor plays an important role in these lung development including epithelial and mesenchymal cell differentiation, surfactant and channel gene expression and septae formation. Over 1400 transcription factor encoded in the mouse genome to date only few are known to be expressed and play a role in the lung. RNA insitu hybridization screening was done to identify transcription factor decoding gene expressed within the branching mouse $^{9}$ especially specific gene FGF.

\section{Materials and methods}

\section{Animals}

All experiments were conducted in accordance with the European Communities Council Directive of 24 November 1986 (86/609/EEC) under the permit T/1571/13.5.09. The protocols were approved by the committee for the Care and Use of Laboratory animals University of Gothenburg, Sweden.

\section{Mouse mutants}

A targeting construct containing a total of $12 \mathrm{~kb}$ of the Foxf1 locus was made from $129 / \mathrm{Sv}$ genomic $\lambda$ clones, in which the forkhead box 
of Foxf1 (from SstI to NotI) was replaced by a PGK-Neo cassette. ${ }^{10}$ The Foxf1 locus was targeted in two ES cell lines: RW4, derived from $129 / \mathrm{SvJ}$, and E14, derived from 129/Ola. Germline transmission was obtained with clones derived from both cell lines and the targeted allele was maintained on mixed 129/Ola-C57Bl/6, 129/Ola-CD1, 129/ Sv-CD1 and 129/Sv-C57B1/6 backgrounds.

\section{In situ hybridization and immunohistochemistry}

Antisense RNA probes were synthesized by In vitro transcription with T3 or T7 RNA polymerase (Takara), according to manufacturer's instructions, using Digoxigenin-11-UTP (Roche). In situ hybridizations of cryosections, Henrique et al., ${ }^{11}$ was performed with digoxigenin-labeled antisense RNA probes. For Foxf1, a 410bp fragment (nt 699-1110 of the mouse cDNA) was used to generate the sense and the antisense probe. ${ }^{11}$ For SP-C, a 163bp fragment (nt 1022 1185 of the mouse cDNA) was used to generate the sense and the antisense probe. ${ }^{12}$ Plasmids used to generate probes for Foxfl was kindly provided by Dr. C. Betsholtz (Goteborg University, Sweden) and for SP-c by Dr. J. Piatigorsky (National Eye Institute, Bethesda, Maryland). The authenticity of PCR products and cDNA clones were verified by DNA sequencing. No signals were observed when control sense probes were used. Non-Radioactive in situ hybridizations of cryosections were performed as described Mahlapuu et al. ${ }^{12}$ The experiments were repeated three times. Sections were analyzed with an Eclipse E800 microscope (Nikon, Japan) fitted with an Infinity 1 digital camera (Lumenera, Canada). Images were captured using the camera software and assembled in Adobe Photoshop.

\section{Results}

We used E13.5 lungs for screening for several reasons. First, epithelial branching has a strict genetic control including transcriptional regulation happens in E13.5 stage. ${ }^{5}$ Second, fibroblast growth factor (FGF), sonic hedgehog (SHH), and bone morphogenetic protein (BMP) signaling pathways are all active in the E13.5 stage. Third, E13.5 is a stage when active patterning of the epithelium and mesenchyme occurs. These broad patterns then serve as blueprints for fine-scale cellular differentiation that occurs at later stages of lung development. Finally, we chose to carry out an in situ hybridization, as this techniques helps in the expression of gene in the subsets of cells in the lung. We determined that E13.5 is the latest, and, therefore, most advanced stage when distinct patterns can still be consistently discerned from tissue section Insitu hybridization. We are interested in genes that play a role in the morphogenesis in the lung.

In order to investigate the distribution of FREAC-1 expression during organogenesis the Foxfl Probe and the highest Foxf1 expression level is seen in the developing lung and evident already at 13dpi (Figure 1).

\section{SP-C probe}

SP-C mRNA was done on the both wild type and the Foxf1 (f1/-) and the comparative number of the cells expressed had been given in the (Table 1). The SP-C was easily detectable in 13dpi mouse lung tissue using a sense cRNA probe (Figure 2). SP-C mRNA was present is more in the WT than the Foxfl (f1/-) in the epithelial cells lining the distal portions of the prealveolar ducts (Figure 3). SP-C mRNA was evenly distributed in all the epithelial cells of distal portion of the tubules and was concentrated in the apical portion of epithelial cells here also more in the WT than the Foxf1 (f1/-) (Figure 4). All the distal portions of the prealveolar tubules that contained SP-C mRNA were rounded.

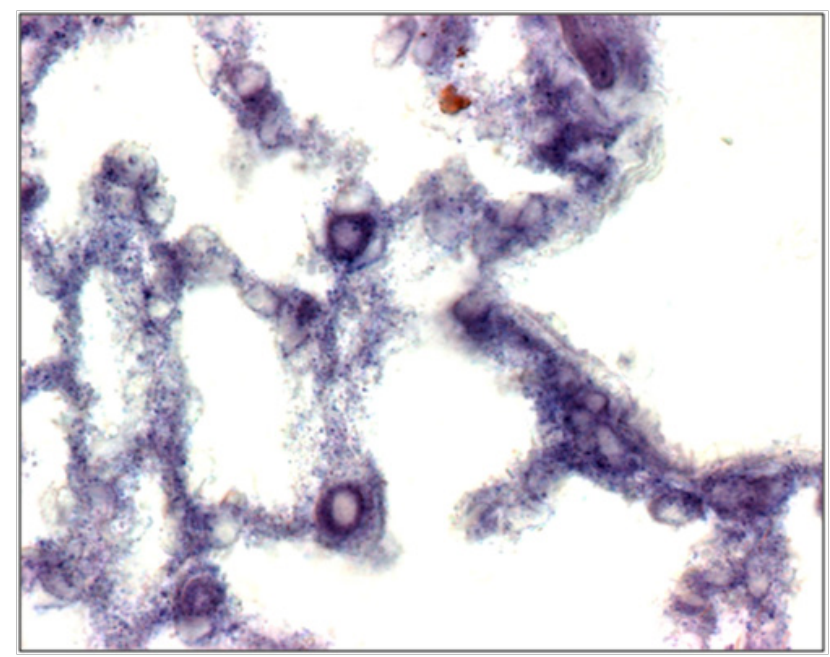

Figure I In situ hybridization analysis of Foxfl expression in mouse lungs. In I3-dpi (A) Foxfl expression is observed in mesenchymal cells especially in the cytoplasm.

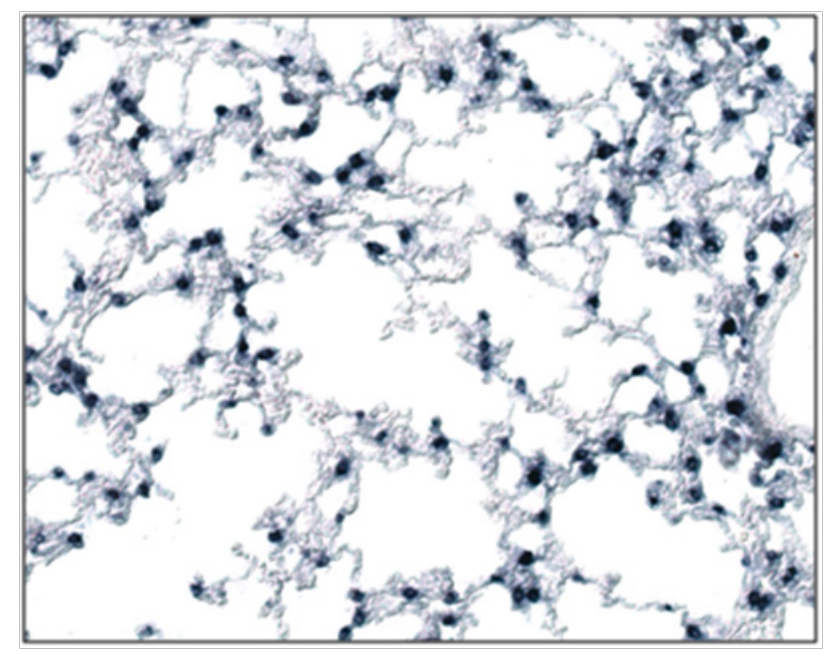

Figure 2 In situ hybridization analysis of SP-C expression in mouse lungs.

In I3-dpi (a) SP-C expression is observed in mesenchymal cells especially in the cytoplasm of the Foxfl ( $\mathrm{fl} /-$ ).

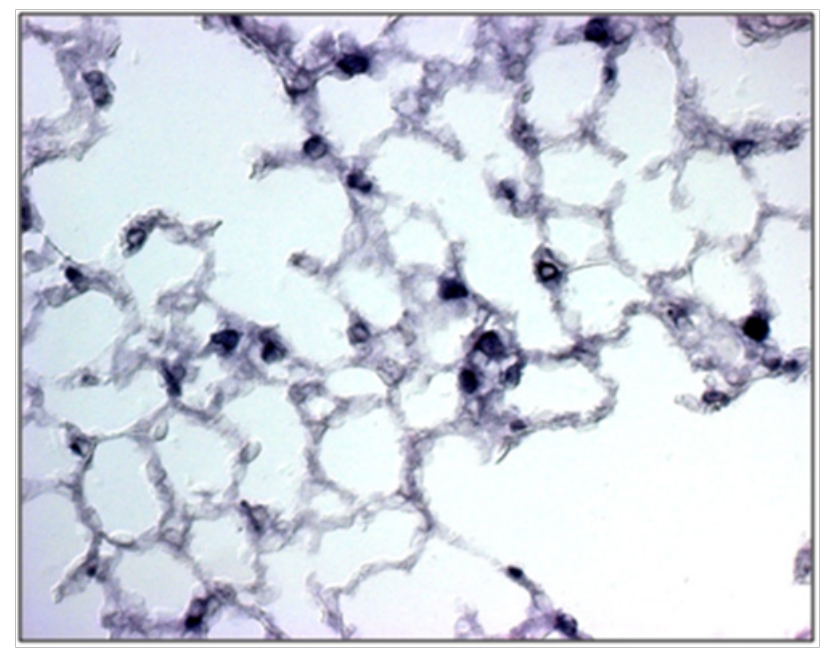

Figure 2b SP-C expression is observed in mesenchymal cells especially in the cytoplasm of the Foxfl ( $\mathrm{fl} /-$ ). 
Table I Insitu Hybridization for SP C probe in lung tissue section

\begin{tabular}{llll} 
Wild type & & & \\
\hline $\begin{array}{l}\text { No. of positive cells in tissue } \\
\text { section I/microscopic area(40x) }\end{array}$ & $\begin{array}{l}\text { No. of positive cells in tissue } \\
\text { section 2/microscopic area(40x) }\end{array}$ & $\begin{array}{l}\text { No. of positive cells in tissue } \\
\text { section 3/microscopic area(40x) }\end{array}$ & $\begin{array}{l}\text { Average no. of positive cells/ } \\
\text { microscopic area (40x) }\end{array}$ \\
\hline 30 & 27 & 17 & 25 \\
27 & 17 & 25 & 23 \\
32 & 26 & 25 & 28 \\
26 & 17 & 22 & 22 \\
23 & 28 & 18 & 23 \\
21 & 25 & 26 & 24 \\
27 & 13 & 23 & 21 \\
15 & 25 & 16 & 29 \\
26 & 27 & 17 & 23 \\
25 & 23 & 21 & 23 \\
\hline
\end{tabular}

Foxfl (fl/-)

\begin{tabular}{|c|c|c|c|}
\hline $\begin{array}{l}\text { No. of positive cells in tissue } \\
\text { section I/microscopic area(40x) }\end{array}$ & $\begin{array}{l}\text { No. of positive cells in tissue } \\
\text { section } 2 / \text { microscopic area( } 40 x)\end{array}$ & $\begin{array}{l}\text { No. of positive cells in tissue } \\
\text { section } 3 / \text { microscopic area( } 40 x)\end{array}$ & $\begin{array}{l}\text { Average no. of positive cells/ } \\
\text { microscopic area }(40 x)\end{array}$ \\
\hline 16 & 28 & 15 & 20 \\
\hline 14 & 18 & 19 & 17 \\
\hline 18 & 18 & 19 & 18 \\
\hline 20 & 22 & 10 & 17 \\
\hline 15 & 18 & 8 & 14 \\
\hline 12 & 22 & 22 & 19 \\
\hline 12 & 18 & 16 & 15 \\
\hline
\end{tabular}

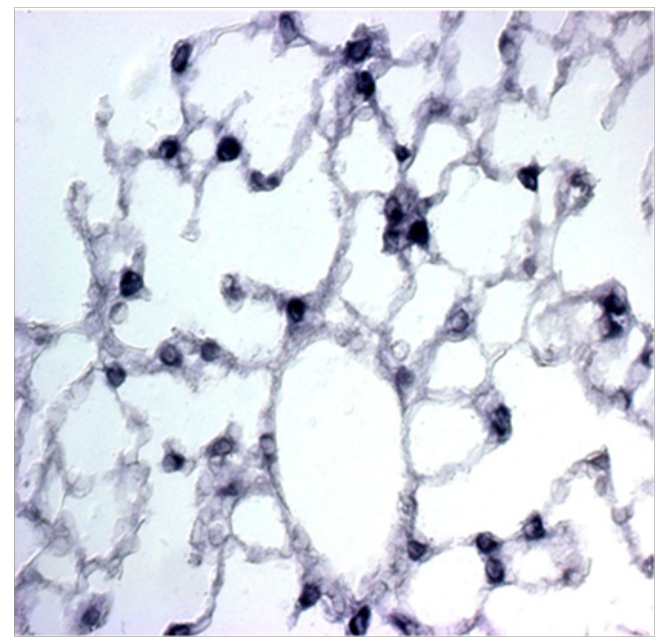

Figure 3 In situ hybridization analysis of SP-C expression in mouse lungs. In I3-dpi (a) SP-C expression is observed in mesenchymal cells especially in the cytoplasm of the Foxfl (fl/-).

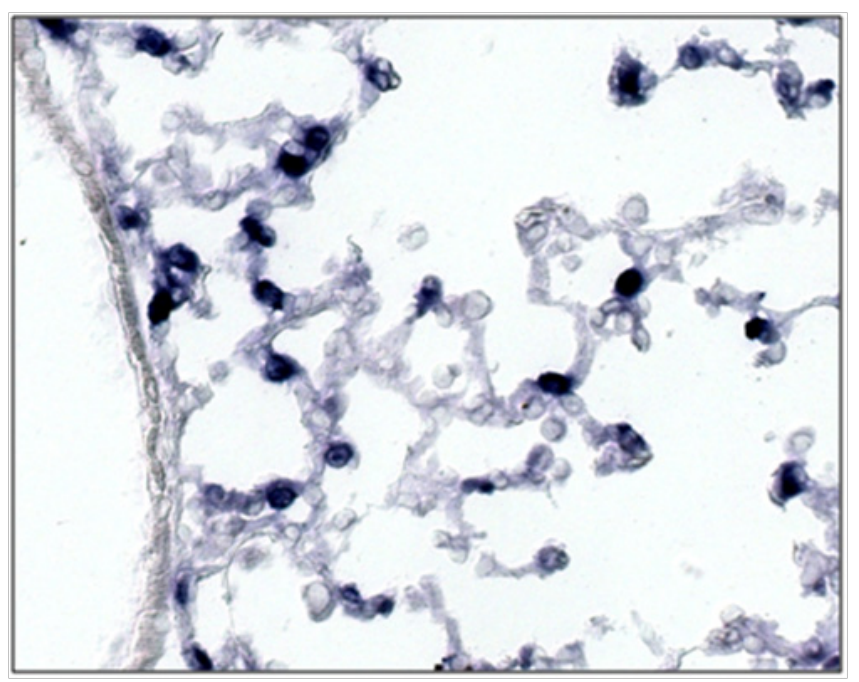

Figure $3 \mathbf{b}$ SP-C expression is observed in mesenchymal cells especially in the cytoplasm of the Foxfl (fl/-). 


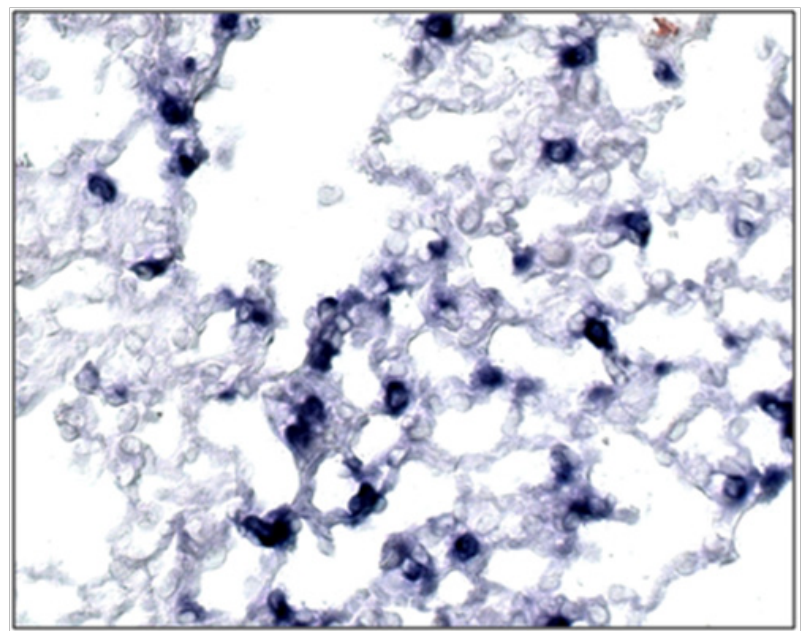

Figure 4 In situ hybridization analysis of SP-C expression in mouse lungs. In I3-dpi (a) SP-C expression is observed in mesenchymal cells especially in the cytoplasm of the Foxfl (fl/-).

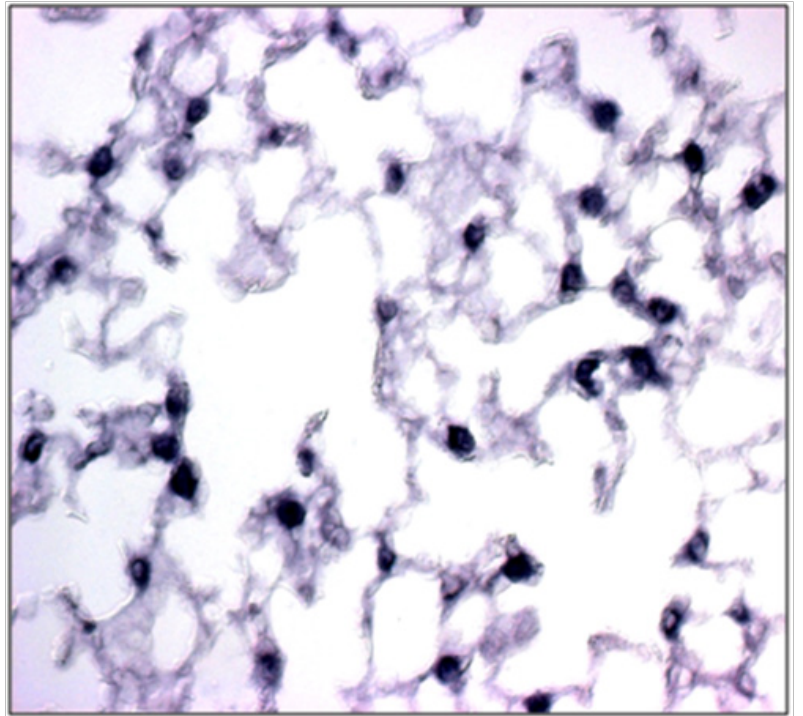

Figure 4b SP-C expression is observed in mesenchymal cells especially in the cytoplasm of the Foxfl ( $\mathrm{fl} /$-).

\section{Discussion}

The Foxf1 protein contains two independent transcriptional activation domains, one located in the $\mathrm{C}$-terminal end of the protein and the other one is central part close to the fork head DNA-binding domain. In transient transfection assays the activation domain in the $\mathrm{C}$-terminus is functional in all cell lines tested and we therefore refer to it as the general activation domain. The central part of the Foxf1 protein, on the other hand, functions as a potent activation domain in two lung-derived cell lines, but is completely inactive in all other cell lines tested. Although results from transformed are more or less dedifferentiated cell lines have to be interpreted with caution, these results do tell us that the function of the activation domain located in the central part of Foxf1 depends on cellular context. Its working mechanism therefore appears to differ from that of most other activation domains and is likely to involve specific adapter or co-activator proteins rather than direct interactions with ubiquitous components of the transcriptional machinery. It should also be noted that the two cell lines in which the cell-type-specific activation domain is active are derived from lung-the organ where the highest expression levels for Foxf1 are found while the non-permissive cell lines are all derived from tissues that do not express Foxfl. Few other examples of cell-type-specific activation domains have been described. The forkhead protein most closely related to Foxf1. Like Foxf1 gene is also interrupted by a single intron ${ }^{13}$ and when the locations of the introns are marked in the amino acid sequences it becomes evident that the genomic organization has been conserved during evolution. The two genes apparently originated through a duplication of a common ancestor, but are today found on different chromosomes. ${ }^{13,14}$ The expression pattern of Foxfl during fetal development suggests that activation of Foxfl transcription may be a primary response to such an epithelium-to-mesenchyme signal.

\section{SP-C}

We detected significant amounts of SP-C mRNA in mouse lung tissue at 13dpi, the earliest time point examined in the present study. Differentiated alveolar type II cells are first observed in mouse lung tissue at approximately 26 days of gestation. ${ }^{15}$ Whitsett et al., ${ }^{15}$ Liley et al. ${ }^{16}(25)$ detected SP-C mRNA in human fetal lung tissue at $13 \mathrm{wk}$ of gestation, i.e., many weeks before the commencement of alveolar type II cell differentiation, which begins at approximately 24 wk of gestation in the human. Schellhause et al. ${ }^{17}$ detected SP-C mRNA in rat fetal lung tissue on day 17 of gestation, the earliest time point examined in their study; differentiated alveolar type II cells are first detected on day 19 of gestation in the rat (term=21days). Together, the results of studies in the human, rat, and rabbit species are suggestive that the expression of SP-C mRNA is not temporally linked to the morphologic differentiation of alveolar type II cells, i.e., to the appearance of intracellular lamellar bodies. The results of the ribonuclease protection assay showed that the SP-C mRNA content of mouse lung tissue increases gradually from 13 dpi to term with a slight decrease in relative concentration apparent in neonatal and adult mouse lung tissue. Our results using Insitu hybridization are also suggestive that the levels of SP-C mRNA present in mouse lung tissue increase with advancing gestational age. Also, the intensity of SP-C mRNA hybridization within alveolar type II cells of adult mouse lung tissue was less than detected in alveolar type II cells in day 31 rabbit fetal lung tissue. Whitsett et al., ${ }^{15}$ Liley et al. ${ }^{16}$ have detected a gradual increase in SP-C mRNA levels in human fetal lung tissue over the period of 16 to $24 \mathrm{wk}$ of gestation. However, the levels of SP-C mRNA present in human fetal lung tissue at $24 \mathrm{wk}$ of gestation were not as great as the SP-C mRNA levels present in adult human lung tissue. Schellhause et al. ${ }^{17}$ found that SP-C mRNA levels increased in rat fetal lung tissue with increasing gestational age and reached a maximum in adult lung tissue. Thus, the developmental pattern of SP-C mRNA induction in the mouse differs from that observed in the human and rat species.

We detected SP-C mRNA in every epithelial cell of the rounded, distal portions of the prealveolar ducts of rabbit fetal lung tissue atdays 13 and 21 dpi. Starting on day 24 of gestation, a few cells in the distal, rounded portion of the prealveolar ducts contained smaller amounts of SP-C mRNA than the majority of epithelial cells in this location. This trend was also apparent on day 26 of gestation, and by day 27 of gestation only a fraction of the epithelial cells contained SP-C mRNA. Thereafter in development, only cells with anatomical characteristics of alveolar type II cells contained SP-C mRNA. Thus, our data are suggestive that the precursor to alveolar type I and type II epithelial cells present early in lung development contains SP-C mRNA and, as alveolar type I cells differentiate starting on day 24 of gestation, they no longer express SP-C mRNA. In contrast, alveolar type II cells continue to express SP-C mRNA throughout the remainder of development. 


\section{Conclusion}

By utilizing the Insitu hybridization techniques, we performed the expression of Foxfl and SP-C protein that show patterned expression in the pseudoglandular stage mouse lung. Many of the transcription factors that we identified have not been previously implicated in lung development. Furthermore, characterization of the expression pattern of some of these genes, such as Grhl2 and Tbx3, has led to consideration of other members of the gene families and their expression in lung. We envision that our findings will promote future studies by: providing additional genes to be investigated for their role in lung development; increasing nodes of the transcriptional circuits that act either upstream or downstream of known factors; and suggesting putative markers for specialized cell populations in the lung at later stages of development and maturation. Going beyond development, our dataset may add value to lung disease research such as the studies of lung cancer. Recent investigations of lung cancer have revealed strong correlations between patient survival rates and misexpression of several of the transcription factors and the surfactant protein highlighted in our screen. For example, increased nuclear localization of ID2 or increased expression of FOXM1, HIF1A, SNAI1, or HOXA9 is associated with higher mortality rates. ${ }^{18-22}$ Thus, our dataset may suggest candidate biomarkers for the correct development of the lungs.

\section{Acknowledgements}

None.

\section{Conflict of interest}

The author declares no conflict of interest.

\section{References}

1. Warburton D, El-Hashash A, Carraro G, et al. Lung organogenesis. Curr Top Dev Biol. 2010;90:73-158.

2. Maeda Y, Davé V, Whitsett JA. Transcriptional control of lung morphogenesis. Physiol Rev. 2007;87(1):219-244.

3. Lü J, Qian J, Izvolsky KI, et al. Global analysis of genes differentially expressed in branching and non-branching regions of the mouse embryonic lung. Dev Biol. 2005;273(2):418-435.

4. Domyan ET, Sun X. Patterning and plasticity in development of the respiratory lineage. Dev Dyn. 2011;240(3):477-485.

5. Metzger DE, Stahlman MT, Shannon JM. Misexpression of ELF5 disrupts lung branching and inhibits epithelial differentiation. Developmental Biology. 2008;320(1):149-160.

6. Rawlins EL, Hogan BL. Epithelial stem cells of the lung: privileged few or opportunities for many. Development. 2006;133(13):2455-2465.
7. Whitsett JA, Weaver TE. Hydrophobic surfactant proteins in lung function and disease. N Engl J Med. 2002;347(26) 2141-2148.

8. Mund SI, Stampanoni M, Schittny JC. Developmental alveolarization of the mouse lung. Dev Dyn. 2008;237(8):2108-2116.

9. Metzger DE, Xu Y, Shannon JM. Elf5 is an epithelium-specific, fibroblast growth factor-sensitive transcription factor in the embryonic lung. Dev Dyn. 2007;236(5):1175-1192.

10. Mahlapuu M, Ormestad M, Enerbäck S, et al. The forkhead transcription factor Foxf1 is required for differentiation of extraembryonic and lateral plate mesoderm. Development. 2001;128(2):155-166.

11. Henrique D, Adam A, Myat A, et al. Expression of a Delta homologue in prospective neurons in the chick. Nature. 1995;375(6534):787-790.

12. Mahlapuu M, Pelto-Huikko M, Aitola S, et al. FREAC-1 contains a cell type-specific transcriptional activation domain and is expressed in epithelialmesenchymal interfaces. Dev Biol. 1998;202(2):183-195.

13. Blixt $\AA$, Mahlapuu M, Darnfors C, et al. The two-exon gene of forkheadrelated activator 2 (FREAC-2) has a TATA-less promoter. Genomics. 1998;53(3):387-390.

14. Larsson C, Hellqvist M, Pierrou S, et al. Chromosomal localization of six human forkhead genes, freac- $1,-3,-4,-5,-6$, and -8 . Genomics. 1995;30(3):464-469.

15. Whitsett, Weaver 1C. Glucocorticoid enhances surfactant proteolipid Phe and pYai synthesis and RNA in fetal lung. Journal of Biological Chemistry. 1998;262(32):15618-15623.

16. Liley HG, Ballard PL. Regulation of messenger RNA: $s$ for the hydrophobic surfactant proteins in human lung. Journal of Investigation. 1998;83(4):1191-1197.

17. Schellhase DE, Ernrie PA, Fisher JH, et al. Ontogeny of surfactant apoproteins in the rat. Pediatric Research. 1989;26(3):167-174.

18. Choi JS, Zheng LT, Ha E, et al. Comparative genomic hybridization array analysis and real-time PCR reveals genomic copy number alteration for lung adenocarcinomas. Lung. 2006;184(6):355-362.

19. Rauch T, Wang Z, Zhang X, et al. Homeobox gene methylation in lung cancer studied by genome-wide analysis with a microarray-based methylated $\mathrm{CpG}$ island recovery assay. Proc Natl Acad Sci U S A. 2007;104(13):5527-5532.

20. Gialmanidis IP, Bravou V, Amanetopoulou SG, et al. Over expression of hedgehog pathway molecules and FOXM1 in non-small cell lung carcinomas. Lung Cancer. 2009;66(1):64-74.

21. Hung JJ, Yang MH, Hsu HS, et al. Prognostic significance of hypoxiainducible factor-1alpha, TWIST1 and Snail expression in resectable non-small cell lung cancer. Thorax. 2009;64(12):1082-1089.

22. Rollin J, Bléchet C, Régina S, et al. The intracellular localization of ID2 expression has a predictive value in non small cell lung cancer. PLoS One. 2009;4(1):4158-4165. 